Chapter 26

\title{
Vascular Access With or Without Synthetic GOR-TEX
}

\author{
Seyed Reza Mousavi
}

Additional information is available at the end of the chapter

http://dx.doi.org/10.5772/54155

\section{Introduction}

Ownership of dialysis facilities has shifted over the past decade. Where nonprofit organizations once managed these facilities, large companies now have control. Shareholders of these companies are primarily interested in the profitability of their investments rather than the well-being of the patients being treated. Market forces rather than scientific inquiry now drive much of the research related to end-stage renal disease (ESRD). These developments have created ethical dilemmas for physicians. This editorial describes some of these ethical challenges and asserts that the doctor-patient relationship and its ethical imperatives are more important than company profit and loss statements.

\section{Dialysis}

\subsection{History}

Gaining proper access to the human circulatory system is required to transport blood from the patient to the artificial kidney and back again during hemodialysis. In 1943, Dr. Willem J. Kolff first encountered the problem of vascular access for hemodialysis [1]. After 34 days of puncturing readily accessible blood vessels, he failed to enter the bloodstream. As a consequence, his patient died. The chronic hemodialysis era began almost 20 years later, when Quinton and Scribner introduced the first external arteriovenous shunt constructed of Teflon, which allowed repeated access to the vascular system [2].

In 1966 a major breakthrough in vascular access surgery was achieved with the introduction of the first endogenous fistula by Brescia and Cimino [3, 4]. They created a side-to-side anastomosis between the radial artery and cephalic vein, which ensured functional vascular access. Later, brachiocephalic and transposed brachiobasilic fistulae were constructed, 
followed by the emergence of various prosthetic grafts as an alternative to native fistula. Polytetrafluoroethylene is now the most frequently used graft material. Nevertheless, vascular access-related complications are still often responsible for patient hospitalization, morbidity, and even mortality $[5,6]$. Facility preference and variations in approach to vascular access practice still seem to be major determinants of vascular access success or failure [7].

\subsection{Costs}

Therapy for ESRD patients is expensive and therefore impracticable in some countries. Even some of the more affluent countries do not provide readily accessible free treatment to all renal failure patients; in 2002, 58\% of hemodialysis providers in the United Kingdom had no vacant staffed slots for new patients [8]. In the United States, discussion has been ongoing as to whether the Medicare system, which provides public funding for patients with renal failure, should be modified as post-World War 2 'baby boomers' reach old age $[9,10]$.

Patients should be allowed time off from their jobs for dialysis treatment and extra time off when complications arise. Paid leave for medical treatment requires cooperation from government and employers. [Remark 3]

This chapter sets out our view of the minimum standards required to provide sufficient care for patients with ESRD. These standards may require modification in response to local circumstances or new research data, but we hope that they will help doctors in all countries to argue for sufficient resources for their patients with ESRD.

[Remark 3] Timing

One ethical issue to be discussed involves the timing of dialysis initiation. Traditional indicators for starting dialysis included the presence of signs and symptoms of uremia in combination with pertinent results of biochemical analyses of serum and plasma [13]. However, several observational cohort and case-control studies have suggested that early initiation of dialysis may improve patients' survival, quality of life, and capacity for employment while decreasing complications $[14,15]$. Although such studies were potentially limited by biases related to lead time, patient selection, and referral time, the clinical practice guidelines that were in use in the late 1990s [16] recommended the commencement of dialysis when the directly measured or calculated (estimated) glomerular filtration rate was higher than the values previously targeted for the initiation of dialysis. However, more recent observational data have suggested that starting dialysis early may in fact be harmful [17, 18]. We assert that if early dialysis benefits patients, it should be used, and, by contrast, if it causes harm, it should not be used, in accordance with the principle of nonmaleficence. [Remark 1]

\section{Ethical issues}

\subsection{Fair selection}

Ideally all patients who can benefit from dialysis should be offered it free of charge. However, many countries cannot afford the substantial cost of treating all patients with renal fail- 
ure by dialysis and transplantation [19]. The problem for poorer countries is exacerbated by their higher incidence of renal failure. The aim of patient selection for dialysis should be to use scarce resources to provide maximum benefit. This implies that patients should be selected who are likely to enjoy a good quality of life as a result of treatment. Selection should not be influenced by race, color, creed, caste, or political affiliation. Age per se should not be a selection criterion. Since comorbidities are more likely in elderly patients, the majority of patients selected for dialysis is likely to be younger. However, many elderly patients may also enjoy a good quality of life [20].

If the only way of obtaining regular dialysis is by payment into a private medical insurance system, patients able and willing to pay the costs should be allowed to do so. No 'waiting list' for dialysis should be maintained, nor should any patient be assigned to a therapy known to be inappropriate to meet his or her needs.

\subsection{Patient choice}

Patient preference plays a part in the selection of the dialysis modality, but this is often overridden by other factors, including availability of hemodialysis stations, difficulty with vascular access placement or preservation, and failure of peritoneal dialysis techniques. In addition, a certain proportion of patients presents late as 'uremic emergencies'. Patients must be informed of the advantages and drawbacks of the various available types of dialysis, the prognosis with and without dialysis treatment, and its effect on quality of life so that they can make informed choices on the basis of current information [21, 22]. Treatment decisions should be consistent with the goals of regaining or maintaining active employment and maximizing rehabilitation of patients into society.

[Remark 3]Privacy and patient rights

The processes of diagnosis, consultation, surgery for vascular access, and dialysis treatment should be conducted in ways that protect patient privacy. They should know the identity of the physicians, nurses, and others involved in their care. They are entitled to (i) refuse a recommended type of treatment and be informed of the medical consequences of this action; (ii) consent or decline to participate in research studies after being fully informed of their purpose [22]; (iii) be treated with respect, dignity, courtesy, compassion, and cultural sensitivity; (iv) have any treatment, possible complications, and self-care requirements explained in an understandable manner, with sufficient time to ask questions and receive answers; (v) be allowed to obtain a second opinion for a given treatment and ask for consultation with another physician; (vi) designate relatives or friends to be kept informed of their medical condition; (vii) be informed about names, dosages, indications, and adverse reactions of all prescribed medications [21]; and (viii) be fully informed about the results of laboratory analyses and any tests they undergo. Gardner et al. demonstrated that a well-developed singleitem measure can be appropriate in avoiding common methods variance, which is often a problem with psychological measures that require respondent self-reports of attitudes, beliefs, perceptions, and the like [23]. [Remark 4] Notwithstanding this limitation, we suggest that patients' beliefs about their illness and treatment are important for their sense of (global) autonomy and self-esteem. Correlations have been identified between appropriate medi- 
cal management, time on dialysis, and positive self-perception of health with better problem-solving ability and higher autonomy, but lower sociotropic personality styles. [Remark 5]

\subsection{Loss of autonomy}

A few studies have been published on the number of patients who decided to withdraw from dialysis once treatment was initiated [11, 12]. [Remark 3] Loss of autonomy was one of the most important reasons for patients to decline dialysis treatment. Patients who chose not to start dialysis were reluctant to give up their freedom and become dependent on medical treatment. They would rather live for a shorter time with more freedom, than live longer with the limitations of a comprehensive treatment such as dialysis. "You are going to die anyway ... and making a trip to the hospital 3 times per week, already being tired and exhausted, and while basically handing your life to others, is such a great deal of effort, I can't do that!" [Remark 6]

\subsection{Quality of life}

One of the most important factors in the choice of treatment in patients with ESRD is quality of life. For many patients, the challenges of living with chronic kidney disease may not prevent them from appreciating the small things in life. Several studies have demonstrated that participation in an exercise program either before or during dialysis can improve quality of life. [Remark 7]

ESRD and its treatments may have a negative impact on quality of life. Nephrologists, like all physicians, must offer their patients all reasonable treatment options. When dialysis is (or is not) a reasonable option is an important ethical issue. The justification for dialysis, like any life-sustaining treatment, is that it prolongs life for a patient who either wishes to live longer or, if cognitively impaired, would (in the opinion of others) be likely to benefit from extension of life.

\section{Case Against Dialysis [Remark 8]}

The following section focuses on patients with advanced dementia or severe and irreversible brain injury who were no longer capable of enjoying life, and hence gained no benefit from dialysis. Guidelines are presented for withholding and withdrawing dialysis and suggestions are offered to help nephrologists avoid causing harm when the patient's family demands that dialysis be performed [24]. [Remark 9]

\subsection{Dialysis refusal}

The Medical Code of Ethics states that patients have the right to refuse medical treatment, and that physicians must accept and respect these decisions. Physicians must guarantee that patients die with dignity while not prolonging or shortening their lives. [Remark 3] Many patients have reported enjoying life and have expressed a desire to live for as long as possible, but not at any price. In one study, however, many patients chose not to start dialysis. 
Most patients who declined dialysis had made the decision before they received information on the treatment and would not have even considered the possibility. These patients indicated that they would have considered treatment when they were younger, in better health, or in severe pain [25].

[Remark 3]In another study, patients decided to discontinue dialysis because of an unacceptable quality of life, depression, and a chronic failure to thrive. Health professionals must support end-of-life decision-making using an ethical decision framework [26].

\subsection{Age-associated decrease in vitality}

Some patients decline dialysis due to an age-associated decrease in vitality. Most patients who declined dialysis reported that they had good lives. However, the discrepancy between their former active lives and their present lives was large. They were incapable of doing many things. Patients noted that they had already had to give up so much in life, and that adding dialysis treatment would be too much. Patients who refused dialysis seemed to be able to face the finiteness of life. They all spoke about the good life they had lived. The serious disruption of life caused by dialysis was unacceptable to them.

A few patients indicated that their overall ability to plan and organize significantly deteriorated with age. As a result, making doctor appointments and arranging to get there in time required too much energy. For most patients, the decision not to pursue dialysis treatment was not well-considered, but in their perception, it was the only option. For those patients with relatively good health, dialysis seemed like a natural decision. Earlier studies showed a worse quality of life in elderly dialysis patients with a higher number of comorbidities and no better survival rates than in patients not undergoing treatment [27-29]. The extent to which elderly patients with poor health status may benefit from this treatment is therefore questionable.

For most patients who declined dialysis, the anticipated loss of autonomy in combination with their age-associated decrease in vitality fueled their refusal. Many patients declined dialysis due to a preference to live from day to day without stress related to illness and treatment. In this study, patients who declined dialysis treatment were mostly men. [Remark 10]

Previous research on the impact of gender on the initiation of dialysis was not available. However, patient withdrawal after treatment initiation has been demonstrated as more common among women than men [30].

\section{Other issues}

\subsection{Social support}

Lack of social support is a significant predictor of mortality, even when a large number of medical variables are controlled.Remark7 Findings of several studies are consistent with the view that social support is an important factor in general health outcome and adjustment to 
chronic and acute illness [31]. Therefore, health care personnel should provide information with honesty to help patients to predict their quality of life and death. Support for the patient and family during the end-of-life period should be multidisciplinary, with clear and timely communication between all members of the team [26]. Understanding the importance of social support at the start of dialysis treatment and its association with survival and wellbeing may have important clinical benefits for patients with ESRD. Clinical practice can then be geared toward the promotion or improvement of patients' support networks. Social support affects health through behavioral, physiological, and psychological mechanisms [32]. Provision of social support can be emotional or tangible. It may involve sharing information or giving advice. The characteristics of ESRD and its treatments are functionally debilitating, affecting social relationships and activities of daily living [33]. Diverse expectations regarding social support between patients and their families and friends may exist. While patients may hope to minimize lifestyle changes despite the restrictions of dialysis, people in their support network may be unaware or unsure of how to cope with the patients' treatment and dietary needs [34]. Feeling socially isolated can induce stress and anxiety, which in turn can produce physiological changes, such as a compromised immune system [28]. If prolonged, these changes could lead to higher morbidity and mortality [35]. Depression has also been associated with lower levels of perceived social support in HD patients [36].

The fact that patients on dialysis require different types of social support has important clinical implications. Clinical care providers may tailor intervention programs to improve social support based on patients' needs, making recommendations to appropriate programs like self-help groups [37] or psychoeducational programs [35-38] designed to promote self-efficacy in coping with dialysis. ESRD patients undergoing dialysis require different types of social support depending on their social environment and severity of the illness. Future studies may provide a longitudinal assessment with several points of data collection, charting possible changes in social support needs since the start of dialysis and their association with survival.

\subsection{Transport and facilities}

For the majority of patients, one-way travel time to receive dialysis should be less than 30 minutes. Ideally, dialysis units and their satellites should be distributed throughout the country. If the nearest unit is far from a patient's home, transportation costs should be fully reimbursed [19]. The dialysis unit should be designed for comfort while waiting as well as during treatment. Communication regarding appointments and medical advice should be readily available.

Approximately half of ESRD patients in developed countries have their own transportation, while the other half are dependent on ambulance or hospital transportation services [20]. Transport should aim to make the dialysis day as short as possible. Each dialysis unit should have nurses trained in renal care, cardiac or respiratory emergencies, and hemodialysis procedures. Each dialysis station can treat up to four ESRD patients per day, but time must be allowed for cleaning and disinfecting the dialysis room and machines. 
Hemodialysis satellites facilities need good road communications and parking facilities. Each dialysis station should have a dialysis machine that measures and controls ultrafiltration, a weighing bed or armchair for the patient, an abundant oxygen supply, and adequate lighting. Testing for viral hepatitis should be performed every 3 months in all patients. HBsAg-positive patients should ideally be dialyzed in a separate dialysis area with dedicated hemodialysis machines, but in practice a dedicated side room and equipment are often used. Patients with hepatitis C virus and HIV carriers can be treated in regular dialysis units, but should be dialyzed with designated hemodialysis machines [22].

\subsection{Dose}

A strong correlation has been demonstrated between hemodialysis dose and patient mortality and morbidity [39]. Hemodialysis adequacy and delivered hemodialysis dose should therefore be assessed regularly, at least once a month, in both adults and children. A stable single-pool Kt/V>1.2 should be provided for at least $90 \%$ of patients. More frequent measurements are necessary when the prescribed hemodialysis dose is not regularly delivered [39].

\section{Author details}

Seyed Reza Mousavi

Shahid Beheshti University Medical Sciences, Tehran, Iran

\section{References}

[1] Kolff WJ, Berk HTH. The artificial kidney: a dialyzer with a great area. Acta Med Scand 1944; 117:121-34

[2] Quinton WE, Dillard D, Scribner BH. Cannulation of blood vessels for prolonged hemodialysis. Trans Am Soc Artif Intern Organs 1960; 104-13

[3] Brescia MJ, Cimino JE, Appel K, Hurwich BJ. Chronic hemodialysis using venipuncture and surgically created arteriovenous fistula. N Eng J Med 1966; 275:1089-92

[4] Kapoian T, Sherman RA. A brief history of vascular access for hemodialysis: an unfinished story. Sem Nephrol 1997; 17:239-45

[5] Powe NR, Jaar B, Furth SL, Hermann J, Briggs W. Septicaemia in dialysis patients: incidence, risk factors and prognosis. Kidney Int 1999; 55:1081-90 
[6] Schwab SJ, Beathard G. The hemodialysis catheter conundrum: hate living with them, but can't live without them. Kidney Int 1999; 56:1-17

[7] Young EW, Dykstra DM, Goodkin DA, Mapes DL, Wolfe RA, Held PJ. Hemodialysis vascular access preferences and outcomes in the DialysisOutcomes and Practices Patterns Study (DOPPS). Kidney Int 2002; 61:2266-71. [Remark 11]

[8] Peters J. Renal Services for Dialysis: Commissioner and Provider Perspectives. National Kidney Research Fund, Peterborough, UK, 2002

[9] Vladeck BC. Learn nothing, forget nothing-the Medicare Commission redux. N Engl J Med 2001; 345:456-8

[10] Charatan F. Bush proposes Medicare reform. BMJ 2003; 326:570

[11] Murtagh F, Cohen LM, Germain MJ. Dialysis discontinuation: quo vadis? Adv Chronic Kidney Dis 2007; 14:379-401

[12] Jager KJ, van Dijk PC, Dekker FW, Stengel B, Simpson K, Briggs JD. The epidemic of aging in renal replacement therapy: an update on elderly patients and their outcomes. Clin Nephrol 2003; 60:352-60.

[13] Hakim RM, Lazarus JM. Initiation of dialysis. J Am Soc Nephrol 1995; 6:1319-28

[14] Bonomini V, Feletti C, Stefoni S, Vangelista A. Early dialysis and renal transplantation. Nephron 1986; 44: 267-71 [Remark 11]

[15] Tattersall J, Greenwood R, Farrington K. Urea kinetics and when to commence dialysis. Am J Nephrol 1995; 15:283-9

[16] National Kidney Foundation. NKFDOQI clinical practice guidelines for peritoneal dialysis adequacy. Am J Kidney Dis 1997; 30:Suppl 2:S67-S136

[17] Beddhu S, Samore MH, Roberts MS, et al. Impact of timing of initiation of dialysis on mortality. J Am Soc Nephrol 2003; 14:2305-12

[18] Traynor JP, Simpson K, Geddes CC, Deighan CJ, Fox JG. Early initiation of dialysis fails to prolong survival in patients with end-stage renal failure. J Am Soc Nephrol 2002; 13:2125-32

[19] Andreucci VE. Rights of CRF failure patients undergoing chronic dialysis therapy. Nephrol Dial Transplant 2004; 19:30-8. [Remark 11]

[20] Lamping DL, Constantinovici N, Roderick P et al. Clinical outcomes, quality of life, and costs in the North Thames Dialysis Study of elderly people on dialysis: a prospective cohort study. Lancet 2000; 356:1543-50

[21] A patient Bill of Rights. AHA Management Advisory 1973. American Hospital Association USA (http://www.aha.org/resource/pbillofrights.asp) 
[22] Transplant Recipients Bill of Rights and Responsibilities. Action Council Executive Committee 1999/2000. American Society of Transplantation and National Kidney Foundation, USA

[23] Gardner DG, Cummings LL, Dunham RB, Pierce JL. Single-item versus multipleitem measurement scales: An empirical comparison. Educ Psychol Meas 1998, 58:898-915

[24] Jeffrey P. Spike Ethical Issues in Dialysis Aaron Spital, Series Editor: Responding to Requests for Dialysis for Severely Demented and Brain Injured Patients Seminars in Dialysis 2007; 20(5):387-90 [Remark 11]

[25] Visser A, Dijkstra GJ, Kuiper D, de Jong P, Franssen C, Gansevoort R, Izaks G, Jager $\mathrm{K}$, Accepting or declining dialysis: considerations taken into account by elderly patients with end-stage renal disease J Nephrol 2009; 22(6):794-9

[26] White Y, Fitzpatrick G. Dialysis: prolonging life or prolonging dying? Ethical, legal and professional considerations for end of life decision making EDTNA ERCA J 2006; 32(2):99-103

[27] Merkus MP, Jager KJ, Dekker FW, Boeschoten EW, Krediet RT. Quality of life in patients on chronic dialysis: self-assessment 3 months after the start of treatment. The Necosad Study Group. Am J Kidney Dis 1997; 29:584-92

[28] Mulder W. Treatment decisions in elderly patients with endstage renal disease [dissertation]. The Netherlands: University of Maastricht; 2002

[29] Murtagh FE, Marsh JE, Donohoe P, Ekbal NJ, Sheerin NS, Harris FE. Dialysis or not? A comparative survival study of patients over 75 years with chronic kidney disease stage 5. Nephrol Dial Transplant 2007; 22:1955-62

[30] Hackett A, Watnick S. Withdrawal from dialysis in end-stage renal disease: medical, social, and psychological issues. Semin Dial 2007; 20:86-90

[31] House JS, Landis KR, Umberson D. Social relationships and health. Science 1988; 241:540-5

[32] Schwarzer R, Knoll N, Rieckmann N. Social support. In: A Kaptein, J Weinman (eds). Health Psychology. Oxford: Blackwell Publishing, 2004, pp. 158-81

[33] Rounds KA, Israel BA. Social networks and social support: living with chronic renal disease. Patient Educ Couns 1985; 7:227-47

[34] Polaschek N. Living on dialysis: concerns of clients in a renal setting. J Adv Nurs 2003; 41:44-52

[35] House JS. Social isolation kills, but how and why? Psychosom Med 2001; 63:273-4

[36] Gençöz T, Astan G. Social support, locus of control, and depressive symptoms in hemodialysis patients. Scand J Psychol 2006; 47:203-8 
[37] Davison KP, Pennebaker JW, Dickerson SS. Who talks? The social psychology of illness support groups. Am Psychol 2000; 55:205-17

[38] Friend R, Singletary Y, Mendell NR, Nurse H. Group participation and survival among patients with end-stage renal disease. Am J Public Health 1986; 76:670-2 39Held PJ, Port FK, Wolfe RA et al. The dose of hemodialysis and patient mortality. Kidney Int 1996; 50:550-6

[39] NKF-DOQI clinical practice guidelines for hemodialysis adequacy. National Kidney Foundation. Am J Kidney Dis 1997; 30:S15-S66 